\title{
Outcomes of Bariatric Surgery in African Americans: an Analysis of the Metabolic and Bariatric Surgery Accreditation and Quality Improvement Program (MBSAQIP) Data Registry
}

\author{
Benedict Y. Hui ${ }^{1} \cdot$ Amanda Roberts $^{1} \cdot$ Kyle J. Thompson ${ }^{2} \cdot$ Iain H. McKillop ${ }^{2} \cdot$ Naresh Sundaresan ${ }^{1} \cdot$ Lauren Poliakin $^{1}$. \\ Selwan D. Barbat ${ }^{1} \cdot$ Timothy S. Kuwada ${ }^{1} \cdot$ Keith S. Gersin $^{1} \cdot$ Abdelrahman Nimeri $^{1}$ (D)
}

Received: 14 May 2020 / Revised: 21 June 2020 / Accepted: 22 June 2020 / Published online: 4 July 2020

(C) Springer Science+Business Media, LLC, part of Springer Nature 2020

\begin{abstract}
Background The incidence of obesity is disproportionally high in African Americans (AA) in the United States. This study compared outcomes for AA patients undergoing Roux-en-Y gastric bypass (RYGB) or sleeve gastrectomy (SG) with non-AA patients.

Methods The MBSAQIP database was reviewed for RYGB and SG patients (2015-2017). Patients were identified as AA or non-AA and grouped to RYGB or SG. Combined and univariate analyses were performed on unmatched/propensity matched populations to assess outcomes.

Results After applying exclusion criteria, 75,409 AA and 354,305 non-AA patients remained. Univariate analysis identified AARYGB and AA-SG patients were heavier and younger than non-AA patients. Overall, AA patients tended to have fewer preoperative comorbidities than non-AA patients with the majority of AA comorbidities related to hypertension and renal disease. Analysis of propensity matched data confirmed AA bariatric surgery patients had increased cardiovascular-related disease incidence compared with non-AA patients. Perioperatively, AA-RYGB patients had longer operative times, increased rates of major complications/ICU admission, and increased incidence of 30-day readmission, re-intervention, and reoperation, concomitant with lower rates of minor complications/superficial surgical site infection (SSI) compared with non-AA patients. For SG, AA patients had longer operative times and higher rates of major complications and 30-day readmission, re-intervention, and mortality, coupled with fewer minor complications, superficial/organ space SSI, and leak.

Conclusion African American patients undergoing bariatric surgery are younger and heavier than non-AA patients and present with different comorbidity profiles. Overall, AAs exhibit worse outcomes following RYGB or SG than non-AA patients, including increased mortality rates in AA-SG patients.
\end{abstract}

Keywords African Americans · Sleeve gastrectomy · Roux-en-Y gastric bypass · Surgical outcomes

Previous Communications This study was presented in part at the 2019 American Society of Metabolic and Bariatric Surgery (ASMBS) Weekend, Chicago, IL.

Abdelrahman Nimeri

Abdelrahman.Nimeri@atriumhealth.org

Benedict Y. Hui

Benedict.Hui@ atriumhealth.org

Amanda Roberts

Amanda.Roberts@atriumhealth.org

Kyle J. Thompson

Kyle.Thompson@atriumhealth.org

Iain H. McKillop

Iain.McKillop@atriumhealth.org

Naresh Sundaresan

Naresh.Sundaresan@atriumhealth.org
Lauren Poliakin

Lauren.Poliakin@atriumhealth.org

Selwan D. Barbat

Selwan.Barbat@atriumhealth.org

Timothy S. Kuwada

Timothy.Kuwada@atriumhealth.org

Keith S. Gersin

Keith.Gersin@atriumhealth.org

1 Atrium Health Weight Management, Section of Bariatric \& Metabolic Surgery, Department of Surgery, Carolinas Medical Center, Atrium Health, Charlotte, NC 28204, USA

2 Division of Research, Department of Surgery, Carolinas Medical Center, Atrium Health, Charlotte, NC, USA 


\section{Background}

Data from the Centers for Disease Control report African Americans (AA) and Hispanics have the highest ageadjusted prevalence of obesity in the United States (46.8\% AAs and $47.0 \%$ Hispanics versus $37.9 \%$ non-Hispanic whites and $12.7 \%$ non-Hispanic Asians) [1]. Despite the differences in obesity rates between races, racial minorities (including AA patients) undergo disproportionately fewer bariatric procedures for treatment of obesity (Roux-en-Y Gastric Bypass [RYGB] or sleeve gastrectomy [SG]) compared with nonHispanic white patients [2-4].

The rise in obesity rates in the United States represents major clinical and financial health challenges. Minorities are disproportionately affected within the obese patient population, often as a result of lower socioeconomic status and/or limited access to healthcare and insurance [5-7]. These factors are likely to contribute to subsequent disparities in both the weight loss options available, as well as the outcomes of such options (including elective bariatric and metabolic surgery). For example, in 2015, Sheka et al. analyzed data from more than100,000 patients in the Metabolic and Bariatric Surgery Accreditation and Quality Improvement Program (MBSAQIP) database and reported AA patients had a higher body-mass index (BMI) at time of operation, concomitant with differences in peri-operative disease burden, longer length of stay, and higher rates of re-admission following either a RYGB or SG procedure. However, the authors used only one year of data, and although they did perform a multivariate analysis, they did not use propensity matching in their analysis [8].

The goals of this study were to utilize the bariatric surgery-specific patient data contained within MBSAQIP to determine the proportion of AA versus non-AA patients undergoing bariatric surgery, analyze the relative differences between pre-operative co-morbidities based on the surgical procedure performed, and determine whether disparities in outcomes between these groups exists using propensity matched patient populations. Primary outcomes were intra-operative outcomes, post-operative complications, and 30-day mortality. Secondary outcomes included patient discharge destination, re-admission, re-intervention, and re-operation.

\section{Methods}

\section{Institutional Assurances}

Our Institutional Review Board has deemed that retrospective analyses of public, anonymized data sets are exempt from review.

\section{Data Source}

Data were accessed from the MBSAQIP Participant Use Files (PUFs) (2015-2017) and queried for patients undergoing laparoscopic RYGB or SG procedures [9]. Exclusion criteria included patients who underwent revisions or conversions, mini-loop gastric bypass, gastric plication, endoscopic therapies, emergency cases, hand-assisted cases, open procedures, or intragastric balloon procedures. Leak rate was determined by filtering the interventions table for intervention related to bariatric surgery due to staple line or anastomotic leak and joined to the main MBSAQIP file by case ID.

\section{Patient Demographics}

Patient demographics and clinical characteristics were compared between AA and non-AA patients to include sex, BMI, and comorbidities at time of their bariatric procedure. For our analysis, several combined variables were generated to allow for clinically significant comparisons to be made between groups. Major complications were defined as acute renal failure, cardiac arrest requiring cardiopulmonary resuscitation, coma $>24 \mathrm{~h}$, cerebral vascular accident (CVA), myocardial infarction (MI), ventilator use $\geq 48 \mathrm{~h}$, pneumonia, progressive renal insufficiency, pulmonary embolism (PE), sepsis, septic shock, unplanned intubation, and/or unplanned admission to the intensive care unit (ICU). A classification of minor complications included wound disruption, surgical site infection (SSI), deep surgical site infection (dSSI), organ space surgical site infection (osSSI), and urinary tract infections (UTI) [10]. Following grouping by race (AA versus non-AA patients), a subgroup analysis was performed based on the type of procedure performed (RYGB or SG).

\section{Statistical Analysis}

Statistical analysis was performed using R Software (V3.4 R Foundation for Statistical Computing, Vienna, Austria). Continuous variables are reported as mean \pm standard deviation, and categorical variables are reported as frequencies and percentages. While performing univariate analyses of continuous variables, paired $t$ test or Wilcoxon's rank-sum tests were employed for parametric (unmatched) and non-parametric (matched) data, respectively. A Chi-square test for categorical variables was used. Covariate matching was performed by nearest neighbor matching algorithm using the MatchIt package [10] with matching being performed based on patient demographics (age, BMI, and race) and comorbid risk factors (diabetes requiring insulin, hypertension (HTN) requiring medication, gastroesophageal reflux disease [GERD], cardiac conditions, hyperlipidemia, history of deep venous thromboembolism [DVT], venous stasis, anticoagulation therapy, renal conditions, previous foregut surgery, smoking status, functional 
independence, mobility status, oxygen usage, obstructive sleep apnea, and chronic steroid usage). Distributions of covariates between control and treatment groups were assessed by absolute mean differences between groups (Fig. 1a and b). A $P$ value of $<0.05$ was considered significant, and a $P$ value of $<0.0001$ was considered highly significant.

\section{Results}

\section{Univariate Analysis of Patient Distribution, Demographics, and Preoperative Observations}

Using the complete MBSAQIP data set (2015-2017), 555,239 cases were identified, from which 429,714 remained after exclusion criteria were applied. Within this data set, there were $75,409(17.55 \%)$ AA and $354,305(82.45 \%)$ non-AA patients (Table 1). Of the AA cohort, $21.78 \%$ underwent a RYGB (versus $28.57 \%$ non-AA) and $78.22 \%$ underwent a SG procedure (versus $71.43 \%$ non-AA) (Table 1). Compared with non-AA patients, AA patients were younger, more likely to be female, and had a higher BMI at time of surgery (Table 1). In considering pre-operative comorbidities, AA and non-AA patients presented with different clinical profiles. Overall, AA patients exhibited fewer comorbidities than non-AA patients with the exception of HTN, history of PE, inferior vena cava (IVC) filter, chronic steroid use, and renal insufficiency/dialysis (Table 1).

\section{Effect of Race on Intraoperative and Post-Operative Observations}

When comparing AA with non-AA patients undergoing a RYGB or SG procedure, operative times were longer for AA patients and AA patients were less likely to have a drain placed (Table 2). Post-operatively, AA patients exhibited increased risk of major complications concomitant with decreased rates of minor complications, and this is reflected in the incidence of the specific post-operative comorbidities described in Table 2.

\section{Roux-en-Y Gastric Bypass Subset Analysis}

When performing a subset analysis based on procedure type, AA patients undergoing RYGB were younger, had higher mean BMIs, and included more females than males compared with non-AA patients (Table 3). When examining matched data sets, only pre-operative BMI remained significantly different $\left(48.41 \pm 9.05\right.$ versus $\left.48.05 \pm 8.83 \mathrm{~kg} / \mathrm{m}^{2}\right)$ (Table 3 ). When using unmatched patient data sets, AA patients exhibited notably different comorbidity profiles compared with non-AA, with AA patients being more likely to have hypertension, renal insufficiency, diabetes (insulin and non-insulin- dependent), require a mobility device, require dialysis, have a history of PE, and have an IVC filter (Table 3). Conversely, non-AA patients were more likely to have GERD, prior PTC, cardiac surgery, history of MI, hyperlipidemia, venous stasis, COPD, and sleep apnea (Table 3). However, the majority of differences in co-morbidities observed between AA and nonAA patients no longer retained significance following data matching and analysis (Table 3 ). The exception to these findings was prior history of DVT $(1.90 \%$ [AA] versus $1.50 \%$ [non-AA]), IVC filter placement (1.46\% [AA] versus $1.19 \%$ [non-AA]), anticoagulation (2.50 [AA] versus 2.08 [non$\mathrm{AA}])$, prior cardiac surgery $(0.80 \%[\mathrm{AA}]$ versus 0.53 [nonAA]), and history of percutaneous transluminal coronary angioplasty (1.66\% [AA] versus $1.36 \%$ [non-AA]) (Table 3).

When considering intra-operative data, operative duration, incidence of drain placement, and conversion to an open approach were higher in AA-RYGB compared with non-AARYGB patients, differences that persisted when analyzing matched patient data sets (Table 4). In evaluating postoperative outcomes, it is of particular note that AA-RYGB patients were more likely to succumb to mortality within 30 days when analyzing unmatched data, but these differences were no longer evident when analyzing matched data. In addition, AA patients were more likely to experience major complications than non-AA patients, and after matching, AARYGB patients had longer operative times, higher major complications (including conversion to open, renal insufficiency (RI), pulmonary embolism (PE), blood transfusion, ICU admission), as well as 30-day readmission, re-intervention, and reoperation rates, and discharge to place other than home (DOTH) compared with non-AA patients (Table 4). Finally, AA-RYGB patients were more likely to experience dehydration, have a drain placed, and undergo a swallow study than non-AARYGB patients, but less likely to have minor complications, superficial SSI, and the anastomosis checked. (Table 4).

\section{Sleeve Gastrectomy Subset Analysis}

A subset analysis of unmatched SG patient data demonstrated that AA-SG patients were younger, had higher mean BMIs, and included more females than males compared with nonAA patients (Table 5). Following propensity matching, AA patients were still younger $(42.18 \pm 10.87$ [AA] versus 42.19 \pm 11.24 years [non-AA]) with higher BMIs $(46.67 \pm 8.40$ [AA] versus $46.46 \pm 8.46$ years [non-AA]) (Table 5). AASG patients had overall fewer co-morbidities when compared with non-AA-SG patients, with non-AA-SG patients having higher rate of GERD, prior PTC, prior cardiac surgery, history of MI, hyperlipidemia, venous stasis, anticoagulation, prior foregut surgery, smoking, COPD, oxygen dependence, and sleep apnea. AA-SG patients did have higher rates of hypertension, renal insufficiency, history of PE, dialysis, as well as presence of IVC filter. As with the RYGB patient subset 
Fig. 1 Covariate balance (Love) plots showing absolute mean difference before (unadjusted; circles) and after (adjusted; squares) propensity score matching using a nearest neighbors algorithm for

(a) Roux-enY gastric bypass

(RYGB) patients and (b) sleeve gastrectomy patients a

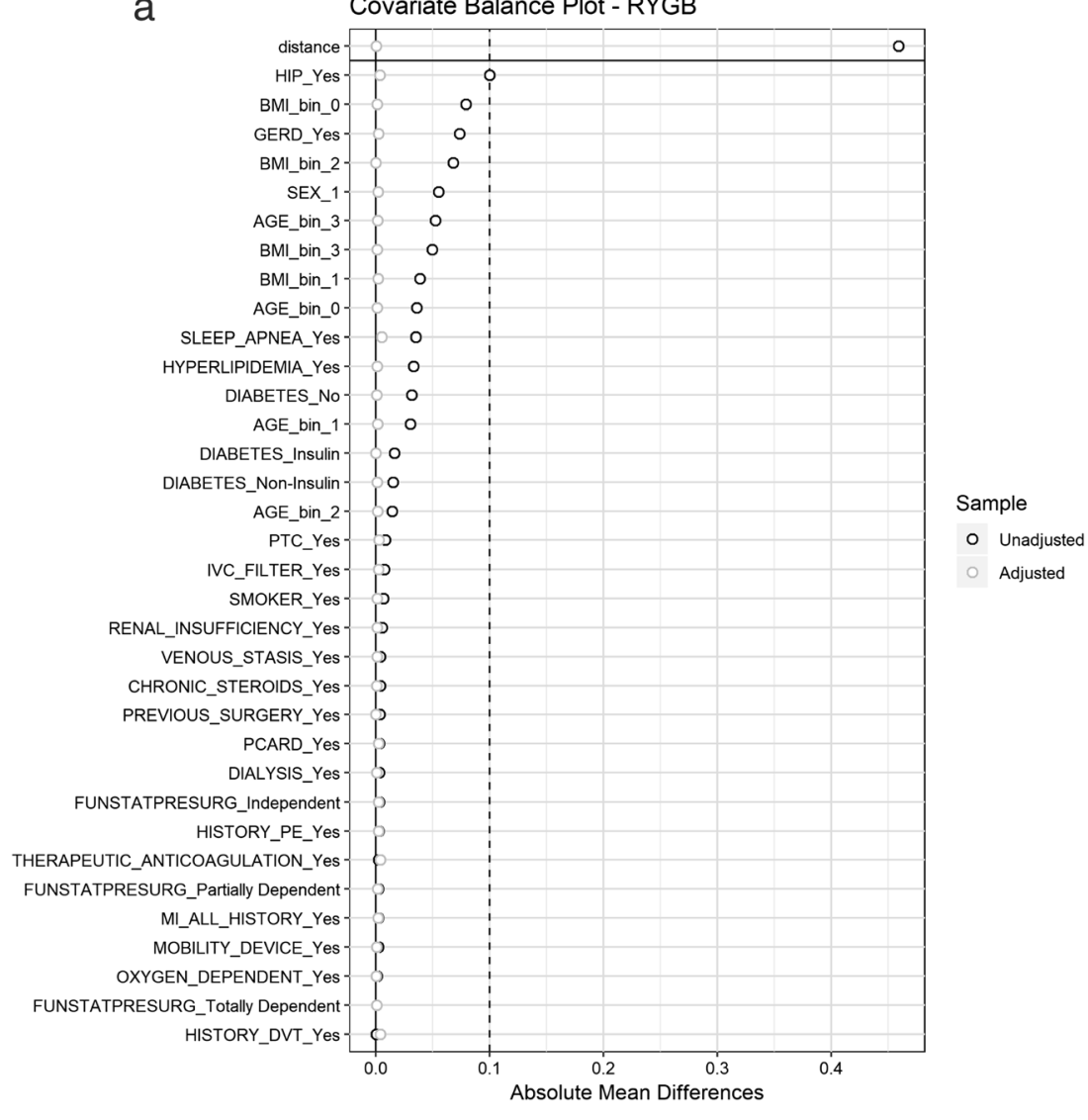

b

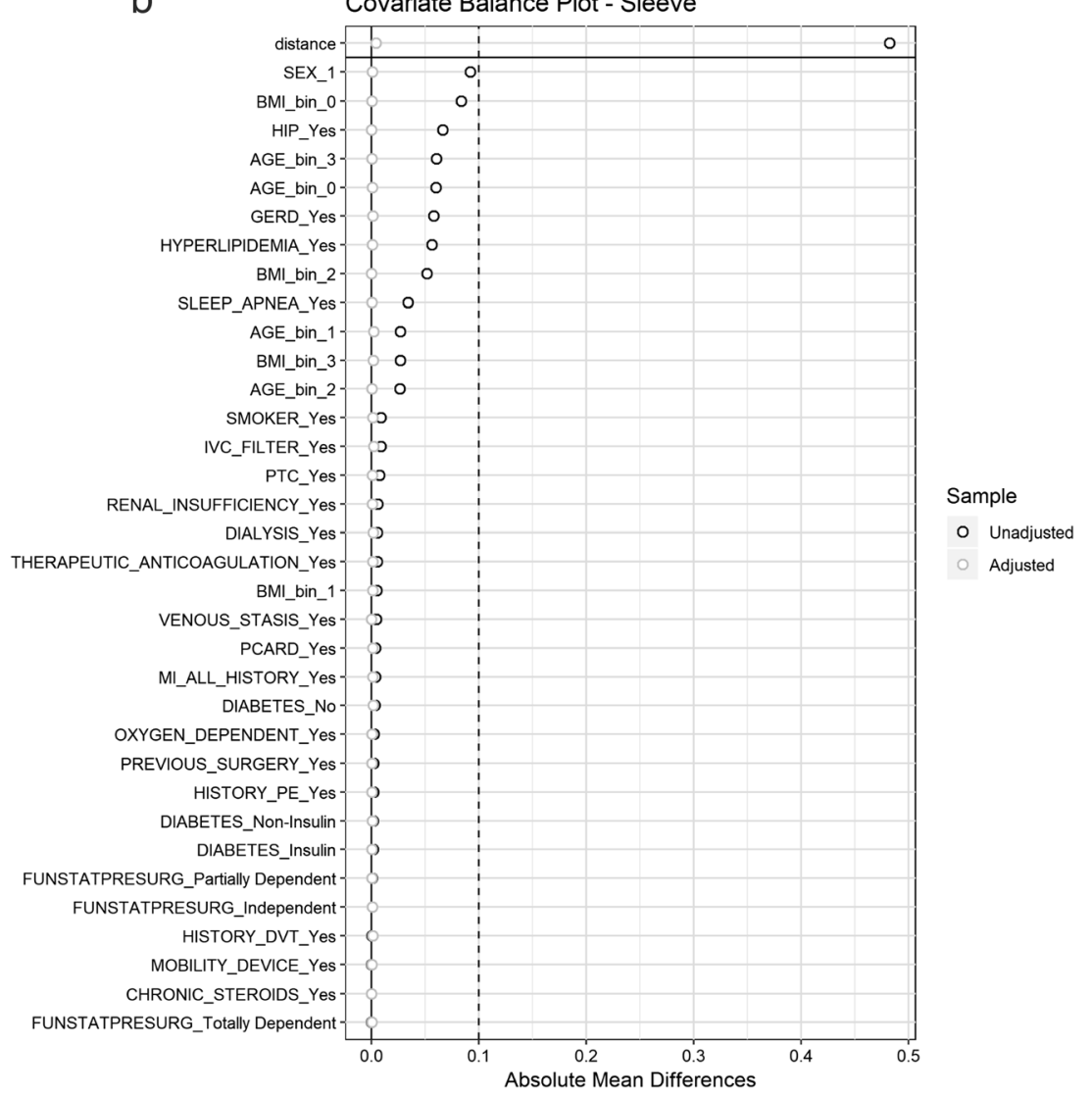


Table 1 Demographics and preoperative comorbidities for African American (AA) vs. nonAfrican American (non-AA) patients undergoing Roux-en-Y gastric bypass (RYGB) or sleeve gastrectomy $(\mathrm{SG})$ procedures

\begin{tabular}{|c|c|c|c|}
\hline & AA & Non-AA & $P$ value \\
\hline \multicolumn{4}{|l|}{ Demographics } \\
\hline$N$ & 75,409 & 354,305 & \\
\hline Frequency $(\%)$ & $17.55 \%$ & $82.45 \%$ & \\
\hline Mean age \pm SD (yrs) & $42.46 \pm 10.93$ & $44.66 \pm 12.13$ & $<0.0001$ \\
\hline Mean pre-op $\mathrm{BMI} \pm \mathrm{SD}\left(\mathrm{kg} / \mathrm{m}^{2}\right)$ & $47.05 \pm 8.58$ & $45.01 \pm 7.73$ & $<0.0001$ \\
\hline Male $(\%)$ & 13.75 & 22.03 & $<0.0001$ \\
\hline Female $(\%)$ & 86.25 & 77.97 & $<0.0001$ \\
\hline RYGB (\%) & 21.78 & 28.57 & $<0.0001$ \\
\hline \multicolumn{4}{|l|}{ Pre-operative comorbidities (\%) } \\
\hline & AA & Non-AA & $P$ value \\
\hline GERD & 25.28 & 31.13 & $<0.0001$ \\
\hline Mobility device & 1.65 & 1.64 & 0.9172 \\
\hline Prior PTC & 1.35 & 2.18 & $<0.05$ \\
\hline Prior cardiac surgery & 0.81 & 1.17 & $<0.0001$ \\
\hline History of MI & 0.99 & 1.35 & $<0.0001$ \\
\hline HTN & 54.25 & 47.29 & $<0.0001$ \\
\hline Hyperlipidemia & 19.47 & 25.04 & $<0.0001$ \\
\hline History of DVT & 1.53 & 1.58 & 0.2828 \\
\hline Venous stasis & 0.61 & 1.10 & $<0.0001$ \\
\hline Renal insufficiency & 1.13 & 0.53 & $<0.0001$ \\
\hline Dialysis & 0.72 & 0.20 & $<0.0001$ \\
\hline Anticoagulation & 2.15 & 2.65 & $<0.0001$ \\
\hline Prior foregut surgery & 1.38 & 1.65 & $<0.0001$ \\
\hline Diabetes insulin & 8.63 & 8.62 & 0.5442 \\
\hline Diabetes non-insulin & 17.91 & 17.75 & 0.5442 \\
\hline Smoker & 7.92 & 8.75 & $<0.0001$ \\
\hline Total dependency & 0.00 & 0.00 & 1.0000 \\
\hline COPD & 1.45 & 1.78 & $<0.0001$ \\
\hline Oxygen dependent & 0.52 & 0.76 & $<0.0001$ \\
\hline History of PE & 1.33 & 1.10 & $<0.0001$ \\
\hline Sleep apnea & 34.86 & 38.80 & $<0.0001$ \\
\hline Chronic steroids & 1.80 & 1.68 & $<0.05$ \\
\hline IVC filter & 1.45 & 0.58 & $<0.0001$ \\
\hline
\end{tabular}

BMI, body mass index; GERD, gastroesophageal reflux disease; PTC, percutaneous transluminal coronary angioplasty; MI, myocardial infarction; HTN, hypertension; DVT, deep vein thrombosis; COPD, chronic obstructive pulmonary disease; PE, pulmonary embolism; IVC, inferior vena cava analysis, many of the initial differences in pre-operative comorbidities between AA and non-AA patients undergoing SG were no longer evidenced when the analyses were performed using matched data (Table 5). The exceptions to these findings were prior history of cardiac surgery/MI, which were higher in the AA cohort compared with the matched non-AA cohort, and incidence of dialysis and history of IVC filter placement which were also higher in AA-SG patients (Table 5).

Perioperatively, the majority of differences identified in unmatched data remained significantly different when analyzing matched data with AA-SG patients being demonstrated to have longer operative times, higher incidence of major complications, including cardiac arrest, RI, PE, unplanned intubation, DVT, 30-day ICU admission, 30-day re-intervention, and 30-day mortality. AA-SG patients also demonstrated higher 30-day readmission, dehydration, and destination other than home compared with non-AA-SG patients (Table 6). Conversely, AA-SG patients had lower rates of minor complications, leak, superficial SSI, and osSSI as compared with non-AA-SG patients (Table 6).

\section{Discussion}

Our analysis using the MBSAQIP dataset identified AA patients undergoing RYGB and SG were younger, more likely to 
Table 2 Outcomes for African American (AA) vs. non-African American (non-AA) patients undergoing Roux-en-Y gastric bypass (RYGB) or sleeve gastrectomy (SG) procedures

\begin{tabular}{|c|c|c|c|}
\hline & AA & Non-AA & $P$ value \\
\hline$N$ & 75,409 & 354,305 & \\
\hline OR time $\pm \mathrm{SD}(\mathrm{min})$ & $88.70 \pm 48.51$ & $85.21 \pm 46.86$ & $<0.0001$ \\
\hline Drain placed & 18.74 & 19.62 & $<0.0001$ \\
\hline Approach converted & 0.17 & 0.15 & 0.1187 \\
\hline Over-sew sleeve & 17.14 & 15.59 & $<0.0001$ \\
\hline Staple line reinforcement & 53.36 & 47.50 & $<0.0001$ \\
\hline Anastomosis checked & 78.31 & 77.91 & $<0.0001$ \\
\hline Discharge other than home & 0.63 & 0.43 & $<0.0001$ \\
\hline Swallow study & 39.91 & 35.37 & $<0.0001$ \\
\hline Drain present at 30 days & 0.22 & 0.18 & $<0.05$ \\
\hline Minor complication & 0.83 & 1.03 & $<0.0001$ \\
\hline Pneumonia & 0.19 & 0.20 & 0.3272 \\
\hline Renal insufficiency & 0.12 & 0.06 & $<0.0001$ \\
\hline Superficial SSI & 0.30 & 0.44 & $<0.0001$ \\
\hline UTI & 0.30 & 0.36 & $<0.05$ \\
\hline Dehydration & 0.47 & 0.22 & $<0.0001$ \\
\hline Major complication & 1.40 & 1.14 & $<0.0001$ \\
\hline Acute renal failure & 0.09 & 0.07 & $<0.05$ \\
\hline CVA & 0.01 & 0.01 & 0.3671 \\
\hline Intra-op. cardiac arrest & 0.05 & 0.04 & 0.1609 \\
\hline Coma $>24 \mathrm{~h}$ & 0.01 & 0.00 & $<0.05$ \\
\hline Deep SSI complication & 0.05 & 0.06 & 0.1881 \\
\hline Intra/post-op MI & 0.02 & 0.03 & $<0.05$ \\
\hline Ventilator $>48 \mathrm{~h}$ & 0.08 & 0.07 & 0.6186 \\
\hline Organ space SSI & 0.15 & 0.21 & $<0.05$ \\
\hline $\mathrm{PE}$ & 0.18 & 0.09 & $<0.0001$ \\
\hline Sepsis & 0.09 & 0.10 & 0.4132 \\
\hline Septic shock & 0.06 & 0.05 & 0.8481 \\
\hline Unplanned intubation & 0.16 & 0.14 & 0.0678 \\
\hline VTE & 0.03 & 0.01 & $<0.05$ \\
\hline DVT requiring treatment & 0.21 & 0.17 & $<0.05$ \\
\hline 30-day ICU admission & 0.80 & 0.65 & $<0.0001$ \\
\hline 30-Day mortality & 0.12 & 0.08 & $<0.05$ \\
\hline 30-Day reoperation & 1.28 & 1.19 & $<0.05$ \\
\hline 30-Day readmission & 4.99 & 3.55 & $<0.0001$ \\
\hline 30-Day intervention & 1.75 & 1.22 & $<0.0001$ \\
\hline PRBC within $72 \mathrm{~h}$ & 0.66 & 0.65 & 0.5876 \\
\hline Bleeding & 0.08 & 0.10 & 0.2289 \\
\hline Leak & 0.07 & 0.12 & $<0.05$ \\
\hline
\end{tabular}

OR, operating room; SSI, surgical site infection; UTI, urinary tract infection; CVA, cerebrovascular accident; MI, myocardial infarction; PE, pulmonary embolism; VTE, venous thromboembolism; DVT, deep vein thrombosis; ICU, intensive care unit; PRBS, packed red blood cell

be female, had higher BMIs, and different preoperative comorbidity profiles compared with non-AA patients. Post-operatively, outcomes for AA patients undergoing RYGB and
SG were worse overall than for non-AA patients, including higher major complications after RYGB and SG, and higher mortality after SG, factors that remained following propensity matching and analysis.

Several studies have addressed outcomes in AA patients previously using a range of available data sets. In a similar manner to our analysis, the Michigan Bariatric Surgery Collaborative compared cohorts of AA and white patients over an eleven-year period. While this study analyzed a smaller dataset than available through MBSAQIP, it is of note that the analysis covers a longer time period (11 years) than the data currently available in MBSAQIP. As reflected in our analysis of unmatched patient data, the Michigan collaborative study reported AA patients had a higher rate of any complication (although no differences in serious complications or mortality were identified), higher rates of emergency department visits, and higher incidence of readmission [11]. When looking at post-operative outcomes Amirian et al. (using MBSAQIP data from 2016) report similar findings to us, whereby AA patients had worse outcomes, including higher postoperative complications, readmission, and reoperations [12], while Tiwari et al., using the University HealthSystem Consortium (UHC) database, also report AA patients were at increased risk of adverse events following a laparoscopic RYGB procedure [13]. Examining mortality after bariatric surgery, Nguyen et al. report AA patients had significantly higher mortality compared with non-Hispanic whites (3.7 vs 2.3 per 1000 procedures, $P<0.01$ ), although their analysis compared non-Hispanic AAs vs non-Hispanic whites and did not include matching and did not compare RYGB and SG separately [14].

When considering the total population who underwent a RYGB or SG procedure, it is of note that AA patients presented preoperatively with higher BMIs and (overall) fewer pre-operative comorbidities than non-AA patients (with the notable exception of HTN, renal insufficiency, dialysis, chronic steroids, IVC filter and history of PE). Similar to our findings, Balasubramanian et al. and Stanford et al. report that obese AA patients have less obesity related comorbidities compared with white patients with equivalent BMIs [15-17]. Similarly, Crawford et al., while examining the General Electric Centricity electronic medical record, found AAs to have lower rates of diabetes and hyperlipidemia compared with whites at similar BMIs of 40 and above, while exhibiting higher rates of hypertension in AAs compared with whites at BMIs of 40 and above [18]. Nunez Lopez et al., while looking at adolescent patients, also found that AA patients were more likely to have a higher BMI as well as higher rates of HTN preoperatively [19]. However, it should be noted AA patients also present for bariatric surgery heavier and at a younger age than non-AA patients suggesting referral/access to care and/or insurance may not fully explain these differences. 
Table 3 Demographics and preoperative comorbidities for African American (AA) vs. nonAfrican American (non-AA) patients undergoing Roux-en-Y gastric bypass (RYGB)

\begin{tabular}{|c|c|c|c|c|c|}
\hline & AA & Non-AA & $P$ value & $\begin{array}{l}\text { Matched } \\
\text { non-AA }\end{array}$ & $P$ value \\
\hline \multicolumn{6}{|l|}{ Demographics } \\
\hline$N$ & 16,423 & 101,226 & & 16,423 & \\
\hline Frequency $(\%)$ & $13.96 \%$ & $86.04 \%$ & & & \\
\hline Mean age \pm SD (yrs) & $43.47 \pm 11.09$ & $45.23 \pm 11.97$ & $<0.0001$ & $43.45 \pm 11.15$ & 0.4980 \\
\hline Mean pre-op $\mathrm{BMI} \pm \mathrm{SD}\left(\mathrm{kg} / \mathrm{m}^{2}\right)$ & $48.41 \pm 9.05$ & $45.75 \pm 7.79$ & $<0.0001$ & $48.05 \pm 8.83$ & $<0.05$ \\
\hline Male & 15.10 & 20.62 & $<0.0001$ & 14.88 & 0.5779 \\
\hline Female & 84.90 & 79.38 & $<0.0001$ & 85.12 & 0.5779 \\
\hline \multicolumn{6}{|l|}{ Pre-operative comorbidities (\%) } \\
\hline GERD & 32.01 & 39.39 & $<0.0001$ & 31.77 & 0.6358 \\
\hline Mobility device & 2.11 & 1.87 & $<0.05$ & 2.02 & 0.5869 \\
\hline Prior PTC & 1.66 & 2.52 & $<0.0001$ & 1.36 & $<0.05$ \\
\hline Prior cardiac surgery & 0.80 & 1.14 & $<0.0001$ & 0.53 & $<0.05$ \\
\hline History of MI & 1.35 & 1.59 & $<0.05$ & 1.14 & 0.0911 \\
\hline HTN & 61.62 & 51.61 & $<0.0001$ & 61.27 & 0.5109 \\
\hline Hyperlipidemia & 26.19 & 29.53 & $<0.0001$ & 26.08 & 0.8114 \\
\hline History of DVT & 1.90 & 1.83 & 0.5641 & 1.50 & $<0.05$ \\
\hline Venous stasis & 0.83 & 1.24 & $<0.0001$ & 0.68 & 0.1261 \\
\hline Renal insufficiency & 1.12 & 0.56 & $<0.0001$ & 0.97 & 0.1933 \\
\hline Dialysis & 0.45 & 0.13 & $<0.0001$ & 0.34 & 0.1137 \\
\hline Anticoagulation & 2.50 & 2.75 & 0.0722 & 2.08 & $<0.05$ \\
\hline Prior foregut surgery & 1.40 & 0.18 & $<0.05$ & 1.37 & 0.8134 \\
\hline Diabetes insulin & 15.39 & 13.74 & $<0.0001$ & 15.36 & 0.9550 \\
\hline Diabetes non-insulin & 22.56 & 21.07 & $<0.0001$ & 22.70 & 0.9950 \\
\hline Smoker & 7.67 & 8.37 & $<0.05$ & 7.79 & 0.6947 \\
\hline Total dependency & 0.38 & 0.30 & 0.0901 & 0.30 & 0.1111 \\
\hline COPD & 1.70 & 2.02 & $<0.05$ & 1.66 & 0.7313 \\
\hline Oxygen dependent & 0.78 & 0.91 & 0.0967 & 0.71 & 0.4806 \\
\hline History of PE & 1.49 & 1.21 & $<0.05$ & 1.25 & 0.0644 \\
\hline Sleep apnea & 40.50 & 44.04 & $<0.0001$ & 39.96 & 0.3112 \\
\hline Chronic steroids & 1.86 & 1.46 & $<0.0001$ & 1.75 & 0.4561 \\
\hline IVC filter & 1.46 & 0.68 & $<0.0001$ & 1.19 & $<0.05$ \\
\hline
\end{tabular}

BMI, body mass index; GERD, gastroesophageal reflux disease; PTC, percutaneous transluminal coronary angioplasty; MI, myocardial infarction; HTN, hypertension; DVT, deep vein thrombosis; COPD, chronic obstructive pulmonary disease; PE, pulmonary embolism; IVC, inferior vena cava
When considering the data presented herein, as well as that published previously, it is imperative to highlight both the nature/size of the datasets available, and the types of analyses performed as they relate to clinical relevance. For example, when considering age and BMI at time of surgery, AA patients were significantly younger and had higher BMIs than non-AA patients whether considering all patients (Table 1, $P<0.001$ ), those undergoing RYGB (Table $3, P<0.0001$ ) or those undergoing SG (Table 5, $P<0.001$ ). However, closer examination of these differences reveals discrepancies in age were of the order of 2-3 years and BMIs of $2-3 \mathrm{~kg} / \mathrm{m}^{2}$, raising questions regarding the clinical significance of such differences relative to other underlying comorbidities that exist between the cohorts and the relevance of such findings to the physician prior to considering procedure options. Similarly, when considering post-operative outcomes, the relative impact of findings such as the significant difference in PRBC use within 72-h evidenced in RYGB patients (1.22 [AA] versus $0.98 \%$ [matched non-AA], $P<0.05$ ), should be weighed against other clinical factors such as incidence of PE, VTE, and 30-day ICU admission, reoperation, intervention, and mortality. Our analysis highlights the importance of using propensity matching when considering the analysis of large datasets such as the MBSAQIP database, and strongly suggests great care should be applied to interpreting the clinical relevance of those differences detected (relative to the size of the datasets being analyzed without matching the groups examined) as well as notable discrepancies that can arise when 
Table 4 Outcomes for African American vs. non-African American (non-AA) patients undergoing Roux-en-Y gastric bypass (RYGB) procedure; univariate and matched data

\begin{tabular}{|c|c|c|c|c|c|}
\hline & $\mathrm{AA}$ & Non-AA & $P$ value & Matched non-AA & $P$ value \\
\hline$N$ & 16,423 & 101,226 & & 16,423 & \\
\hline OR time $\pm \mathrm{SD}$ & $127.25 \pm 58.69$ & $118.60 \pm 53.52$ & $<0.0001$ & $117.14 \pm 51.26$ & $<0.0001$ \\
\hline Drain placed & 29.98 & 27.70 & $<0.0001$ & 27.85 & $<0.0001$ \\
\hline Approach converted & 0.37 & 0.27 & $<0.05$ & 0.18 & $<0.05$ \\
\hline Anastomosis checked & 91.40 & 92.65 & $<0.0001$ & 92.27 & $<0.05$ \\
\hline Discharge other than home & 0.86 & 0.54 & $<0.0001$ & 0.44 & $<0.0001$ \\
\hline Swallow study & 33.34 & 27.78 & $<0.0001$ & 28.04 & $<0.0001$ \\
\hline Drain present at 30 days & 0.20 & 0.29 & $<0.05$ & 0.26 & 0.2981 \\
\hline Minor complication & 1.67 & 1.84 & 0.1257 & 2.01 & $<0.05$ \\
\hline Pneumonia & 0.38 & 0.38 & 0.9264 & 0.35 & 0.6461 \\
\hline Renal insufficiency & 0.23 & 0.09 & $<0.0001$ & 0.09 & $<0.05$ \\
\hline Superficial SSI & 0.77 & 0.91 & 0.0928 & 1.08 & $<0.05$ \\
\hline UTI & 0.47 & 0.51 & 0.4531 & 0.52 & 0.4798 \\
\hline Dehydration & 0.77 & 0.38 & $<0.0001$ & 0.47 & $<0.05$ \\
\hline Major complication & 2.15 & 1.76 & $<0.05$ & 1.64 & $<0.05$ \\
\hline Acute renal failure & 0.17 & 0.11 & $<0.05$ & 0.10 & 0.1008 \\
\hline CVA & 0.02 & 0 & 0.0547 & 0.01 & 0.6547 \\
\hline Cardiac arrest & 0.07 & 0.06 & 0.6717 & 0.06 & 0.8272 \\
\hline Coma $>24 \mathrm{~h}$ & 0.01 & 0 & $<0.05$ & 0 & 0.3173 \\
\hline Deep SSI complication & 0.15 & 0.14 & 0.9028 & 0.13 & 0.6545 \\
\hline Intra/post-op MI & 0.03 & 0.05 & 0.2583 & 0.05 & 0.4053 \\
\hline Ventilator $>48 \mathrm{~h}$ & 0.19 & 0.14 & $<0.05$ & 0.13 & 0.1732 \\
\hline Organ space SSI & 0.33 & 0.36 & 0.5901 & 0.33 & 0.9236 \\
\hline $\mathrm{PE}$ & 0.26 & 0.14 & $<0.05$ & 0.11 & $<0.05$ \\
\hline Sepsis & 0.21 & 0.17 & 0.3504 & 0.17 & 0.4456 \\
\hline Septic shock & 0.13 & 0.11 & 0.3692 & 0.10 & 0.4231 \\
\hline Unplanned intubation & 0.29 & 0.23 & 0.1980 & 0.20 & 0.1171 \\
\hline VTE & 0.01 & 0.01 & 0.9719 & 0.01 & 1.0000 \\
\hline DVT requiring treatment & 0.19 & 0.17 & 0.6100 & 0.17 & 0.6959 \\
\hline 30-Day ICU admission & 1.36 & 1.08 & $<0.05$ & 0.97 & $<0.05$ \\
\hline 30-Day mortality & 0.21 & 0.14 & $<0.05$ & 0.15 & 0.1888 \\
\hline 30-Day reoperation & 2.55 & 2.16 & $<0.05$ & 2.01 & $<0.05$ \\
\hline 30-Day readmission & 7.71 & 5.56 & $<0.0001$ & 5.36 & $<0.0001$ \\
\hline 30-Day intervention & 3.29 & 2.28 & $<0.0001$ & 2.32 & $<0.0001$ \\
\hline PRBC within $72 \mathrm{~h}$ & 1.22 & 1.07 & 0.0838 & 0.98 & $<0.05$ \\
\hline Bleeding & 0.27 & 0.24 & 0.5840 & 0.24 & 0.5827 \\
\hline Leak & 0.09 & 0.10 & 0.5567 & 0.11 & 0.4793 \\
\hline
\end{tabular}

OR, operating room; SSI, surgical site infection; UTI, urinary tract infection; CVA, cerebrovascular accident; MI, myocardial infarction; PE, pulmonary embolism; VTE, venous thromboembolism; DVT, deep vein thrombosis; ICU, intensive care unit; PRBS, packed red blood cell comparing "total datasets" with matched patient datasets [8, 20].

Given the high rates of obesity in the AA population, the impact of obesity on health, and the rates of post-operative complications that currently exist, our data suggest greater availability to bariatric surgery for AA-patients may be an important factor in improving outcomes and decreasing post-operative complication rates. Previous studies report
AAs tend to be less educated about metabolic and bariatric surgery (MBS) and have less weight loss than whites after MBS [21-23]. To fully examine the impact race may have on pre-operative comorbidities and outcomes for bariatric surgery it is important that other factors be considered [24]. For example, significant components in these types of studies may relate to the availability of access to healthcare, and bariatric surgery specifically, as well as socioeconomic, psychological 
Table 5 Demographics and preoperative comorbidities for African American (AA) vs. nonAfrican American (non-AA) patients undergoing sleeve gastrectomy (SG) procedure

\begin{tabular}{|c|c|c|c|c|c|}
\hline & $\mathrm{AA}$ & Non-AA & $P$ value & $\begin{array}{l}\text { Matched } \\
\text { non-AA }\end{array}$ & $P$ value \\
\hline \multicolumn{6}{|l|}{ Demographics } \\
\hline$N$ & 58,986 & 253,079 & & 58,986 & \\
\hline Frequency $(\%)$ & $18.90 \%$ & $81.10 \%$ & & & \\
\hline Mean age $\pm \mathrm{SD}$ (yrs) & $42.18 \pm 10.87$ & $44.44 \pm 12.19$ & $<0.0001$ & $42.19 \pm 11.24$ & $<0.0001$ \\
\hline Mean pre-op $\mathrm{BMI} \pm \mathrm{SD}\left(\mathrm{kg} / \mathrm{m}^{2}\right)$ & $46.67 \pm 8.40$ & $44.71 \pm 7.69$ & $<0.0001$ & $46.46 \pm 8.46$ & $<0.0001$ \\
\hline Male & 13.38 & 22.59 & $<0.0001$ & 13.29 & 0.6747 \\
\hline Female & 86.62 & 77.41 & $<0.0001$ & 86.71 & 0.6747 \\
\hline \multicolumn{6}{|l|}{ Pre-operative comorbidities (\%) } \\
\hline GERD & 23.40 & 29.22 & $<0.0001$ & 23.26 & 0.5724 \\
\hline Mobility device & 1.52 & 1.55 & 0.5987 & 1.47 & 0.5328 \\
\hline Prior PTC & 1.27 & 2.04 & $<0.0001$ & 1.17 & 0.1306 \\
\hline Prior cardiac surgery & 0.81 & 1.18 & $<0.0001$ & 0.68 & $<0.05$ \\
\hline History of MI & 0.89 & 1.26 & $<0.0001$ & 0.77 & $<0.05$ \\
\hline HTN & 52.20 & 45.56 & $<0.0001$ & 52.21 & 0.9675 \\
\hline Hyperlipidemia & 17.59 & 23.24 & $<0.0001$ & 17.49 & 0.6570 \\
\hline History of DVT & 1.43 & 1.48 & 0.3070 & 1.30 & 0.0564 \\
\hline Venous stasis & 0.55 & 1.04 & $<0.0001$ & 0.52 & 0.5766 \\
\hline Renal insufficiency & 1.13 & 0.52 & $<0.0001$ & 1.02 & 0.0549 \\
\hline Dialysis & 0.81 & 0.23 & $<0.0001$ & 0.63 & $<0.05$ \\
\hline Anticoagulation & 2.06 & 2.61 & $<0.0001$ & 1.91 & 0.0601 \\
\hline Prior foregut surgery & 1.37 & 1.59 & $<0.0001$ & 1.40 & 0.6185 \\
\hline Diabetes insulin & 6.75 & 6.58 & 0.1360 & 6.81 & 0.7827 \\
\hline Diabetes non-insulin & 16.62 & 16.43 & 0.1360 & 16.73 & 0.7827 \\
\hline Smoker & 7.99 & 8.90 & $<0.0001$ & 7.84 & 0.3650 \\
\hline Total dependency & 0.43 & 0.44 & 0.6833 & 0.39 & 0.3735 \\
\hline COPD & 1.37 & 1.68 & $<0.0001$ & 1.39 & 0.7840 \\
\hline Oxygen dependent & 0.45 & 0.69 & $<0.0001$ & 0.40 & 0.2095 \\
\hline History of PE & 1.29 & 1.06 & $<0.0001$ & 1.22 & 0.3463 \\
\hline Sleep apnea & 33.28 & 36.70 & $<0.0001$ & 33.24 & 0.8724 \\
\hline Chronic steroids & 1.78 & 1.77 & 0.8221 & 1.78 & 0.9649 \\
\hline IVC filter & 1.45 & 0.55 & $<0.0001$ & 1.22 & $<0.05$ \\
\hline
\end{tabular}

BMI, body mass index; GERD, gastroesophageal reflux disease; PTC, percutaneous transluminal coronary angioplasty; MI, myocardial infarction; HTN, hypertension; DVT, deep vein thrombosis; COPD, chronic obstructive pulmonary disease; PE, pulmonary embolism; IVC, inferior vena cava variables, rural vs urban residency, differences in referral for bariatric surgery, and insurance status, but these are not available for analysis in the MBSAQIP dataset [6, 7, 24-30].

Large data sets, such as MBSAQIP, provide the opportunity to analyze outcomes from multiple sites for surgical procedures. However, using such datasets for these analyses is not without limitations imposed by the nature of retrospective database studies. Specifically, the analyses performed is, by the nature of this type of study, limited by the data available within the MBSAQIP database. For example, MBSAQIP only captures data up to 30-days post-operatively, and thus we were unable to analyze long-term outcomes, such as sustained weight loss, comorbidity resolution, or complications occurring more than 30-days postoperatively. Similarly, MBSAQIP does not include regional data or information regarding insurance or socioeconomic status, and these data may be of particular significance when analyzing historically underserved patients.

Finally, our analyses focused on differences between AA patients compared with all other races (in the United States). Epidemiological data report higher obesity rates in African Americans and Hispanics compared with white, nonHispanics in the United States [1]. Given similarities in socio-economic factors between AAs and Hispanics, and the racial disparities that exist in access to health care, education, and/or insurance [31], it would be of great interest to utilize the MBSAQIP to examine potential similarities and/or differences in underlying comorbidities and perioperative outcomes 
Table 6 Outcomes for African American vs. non-African American (non-AA) patients undergoing sleeve gastrectomy (SG) procedure; univariate and matched data

\begin{tabular}{|c|c|c|c|c|c|}
\hline & $\mathrm{AA}$ & Non-AA & $P$ value & Matched non-AA & $P$ value \\
\hline$N$ & 58,986 & 253,079 & & 58,986 & \\
\hline OR time \pm SD & $77.97 \pm 39.00$ & $71.86 \pm 36.12$ & $<0.0001$ & $71.09 \pm 36.06$ & $<0.0001$ \\
\hline Drain placed & 15.61 & 16.39 & $<0.0001$ & 16.71 & $<0.0001$ \\
\hline Approach converted & 0.12 & 0.10 & 0.1723 & 0.10 & 0.4793 \\
\hline Anastomosis checked & 74.67 & 72.01 & $<0.0001$ & 72.06 & $<0.0001$ \\
\hline Discharge other than home & 0.56 & 0.39 & $<0.0001$ & 0.35 & $<0.0001$ \\
\hline Swallow study & 41.74 & 38.41 & $<0.0001$ & 38.64 & $<0.0001$ \\
\hline Drain present at 30 days & 0.23 & 0.14 & $<0.0001$ & 0.17 & 0.0229 \\
\hline Minor complication & 0.59 & 0.70 & $<0.05$ & 0.72 & $<0.05$ \\
\hline Pneumonia & 0.13 & 0.13 & 0.9685 & 0.13 & 0.7454 \\
\hline Renal insufficiency & 0.08 & 0.04 & $<0.0001$ & 0.04 & $<0.05$ \\
\hline Superficial SSI & 0.17 & 0.25 & $<0.05$ & 0.26 & $<0.05$ \\
\hline UTI & 0.26 & 0.30 & 0.1000 & 0.31 & 0.0786 \\
\hline Dehydration & 0.39 & 0.15 & $<0.0001$ & 0.17 & $<0.0001$ \\
\hline Major complication & 1.19 & 0.89 & $<0.0001$ & 0.90 & $<0.0001$ \\
\hline Acute renal failure & 0.07 & 0.05 & 0.0573 & 0.04 & 0.1068 \\
\hline CVA & 0.01 & 0.01 & 0.9205 & 0.01 & 0.5930 \\
\hline Cardiac arrest & 0.04 & 0.03 & 0.0739 & 0.02 & $<0.05$ \\
\hline Coma $>24 \mathrm{~h}$ & 0.01 & 0.00 & $<0.05$ & 0.00 & 0.1025 \\
\hline Deep SSI complication & 0.02 & 0.03 & 0.3241 & 0.03 & 0.4327 \\
\hline Intra/post-op MI & 0.02 & 0.03 & 0.1317 & 0.02 & 1.0000 \\
\hline Ventilator $>48 \mathrm{~h}$ & 0.05 & 0.05 & 0.9311 & 0.03 & 0.3071 \\
\hline Organ space SSI & 0.10 & 0.15 & $<0.05$ & 0.16 & $<0.05$ \\
\hline $\mathrm{PE}$ & 0.15 & 0.07 & $<0.0001$ & 0.06 & $<0.0001$ \\
\hline Sepsis & 0.06 & 0.07 & 0.2599 & 0.07 & 0.3522 \\
\hline Septic shock & 0.03 & 0.03 & 0.8172 & 0.02 & 0.3034 \\
\hline Unplanned intubation & 0.13 & 0.10 & $<0.05$ & 0.08 & $<0.05$ \\
\hline VTE & 0.03 & 0.01 & 0.0013 & 0.02 & 0.2230 \\
\hline DVT requiring treatment & 0.21 & 0.17 & $<0.05$ & 0.15 & $<0.05$ \\
\hline 30-Day ICU admission & 0.65 & 0.48 & $<0.0001$ & 0.49 & $<0.05$ \\
\hline 30-Day mortality & 0.10 & 0.06 & $<0.05$ & 0.05 & $<0.05$ \\
\hline 30-Day reoperation & 0.93 & 0.81 & $<0.05$ & 0.97 & 0.2946 \\
\hline 30-Day readmission & 4.23 & 2.75 & $<0.0001$ & 2.87 & $<0.0001$ \\
\hline 30-Day intervention & 1.32 & 0.80 & $<0.0001$ & 0.87 & $<0.0001$ \\
\hline PRBC within $72 \mathrm{~h}$ & 0.51 & 0.48 & 0.3230 & 0.43 & 0.0614 \\
\hline Bleeding & 0.03 & 0.04 & 0.3851 & 0.03 & 0.8728 \\
\hline Leak & 0.07 & 0.13 & $<0.0001$ & 0.14 & $<0.05$ \\
\hline
\end{tabular}

OR, operating room; SSI, surgical site infection; UTI, urinary tract infection; CVA, cerebrovascular accident; MI, myocardial infarction; PE, pulmonary embolism; VTE, venous thrombo-embolism; DVT, deep vein thrombosis; ICU, intensive care unit; PRBS, packed red blood cell between $\mathrm{AA}$ and Hispanic patients undergoing bariatric surgery procedures for the treatment of obesity.

\section{Conclusions}

Using the MBSAQIP dataset (2015-2017), we identify that AA patients who undergo a RYGB or SG procedure are younger, heavier, and with different but overall lower comorbidities compared with non-AA patients. Close review of the specifics of these findings suggests care should be taken in interpreting data as it pertains to statistical differences relative to clinical significance. Nevertheless, when using matched patient data, it is of note that we identify AA patients continue to exhibit worse outcomes, including higher rates of major complications in both RYGB and SG patient subsets, and 
higher mortality in SG patients compared with non-AA patients.

\section{Compliance with Ethical Standards}

Conflict of Interest Disclosures Drs. Hui, Roberts, Thompson, Barbat, Sundarsesan, Poliakin, and Mckillop have no conflicts of interest or financial ties to disclose.

Dr. Kuwada is a speaker for BD Bard and WL Gore and a consultant for Medtronic.

Dr. Gersin is a speaker for WL Gore and Medtronic, an investor in Standard Bariatrics, and a consultant for GI dynamics.

Dr. Nimeri is on the speakers' bureau of Medtronic.

Ethical Statement All procedures performed in studies involving human participants were in accordance with the ethical standards of the institutional and/or national research committee and with the 1964 Helsinki declaration and its later amendments or comparable ethical standards. For this type of study, formal consent is not required.

Consent Statement Informed consent does not apply.

\section{References}

1. Prevention, C.f.D.C.a. Available from: https://www.cdc.gov/ obesity/data/adult.html. April 2020

2. Jackson TD, Zhang R, Glockler D, et al. Health inequity in access to bariatric surgery: a protocol for a systematic review. Syst Rev. 2014:3:15

3. Hoffman AB, Myneni AA, Orom H, et al. Disparity in access to bariatric surgery among African-American men. Surg Endosc. 2019;34(6):2630-2637. https://doi.org/10.1007/s00464-01907034-z

4. Worni M, Guller U, Maciejewski ML, et al. Racial differences among patients undergoing laparoscopic gastric bypass surgery: a population-based trend analysis from 2002 to 2008. Obes Surg. 2013;23(2):226-33.

5. Kirby JB, Kaneda T. Unhealthy and uninsured: exploring racial differences in health and health insurance coverage using a life table approach. Demography. 2010;47(4):1035-51.

6. Ofori A, Keeton J, Booker Q, et al. Socioecological factors associated with ethnic disparities in metabolic and bariatric surgery utilization: a qualitative study. Surg Obes Relat Dis. 2020;16:786-95.

7. Hecht LM, Pester B, Braciszewski JM, et al. Socioeconomic and racial disparities in bariatric surgery. Obes Surg. 2020;30:2445-9.

8. Sheka AC, Kizy S, Wirth K, et al. Racial disparities in perioperative outcomes after bariatric surgery. Surg Obes Relat Dis. 2019;15(5): 786-93.

9. Barbat SD, Thompson KJ, Raheem E, et al. Bariatric surgery outcomes when assisted by fellows and residents: an MBSAQIP analysis of 477,670 patients. Surg Obes Relat Dis. 2020;16(5):651-7.

10. Dugan N, Thompson KJ, Barbat S, et al. Male gender is an independent risk factor for patients undergoing laparoscopic sleeve gastrectomy or roux-en-Y gastric bypass: an MBSAQIP(R) database analysis. Surg Endosc. 2020;In Press.

11. Wood MH, Carlin AM, Ghaferi AA, et al. Association of race with bariatric surgery outcomes. JAMA Surg. 2019;154(5):e190029.

12. Amirian H, Torquati A, Omotosho P. Racial disparity in 30-day outcomes of metabolic and bariatric surgery. Obes Surg. 2020;30(3):1011-20.
13. Tiwari MM, Goede MR, Reynoso JF, et al. Differences in outcomes of laparoscopic gastric bypass. Surg Obes Relat Dis. 2011;7(3): 277-82.

14. Nguyen GC, Patel AM. Racial disparities in mortality in patients undergoing bariatric surgery in the U.S.A. Obes Surg. 2013;23(10): 1508-14.

15. Balasubramanian BA, Garcia MP, Corley DA, et al. Racial/ethnic differences in obesity and comorbidities between safety-net- and non safety-net integrated health systems. Medicine (Baltimore). 2017;96(11):e6326.

16. Stanford FC, Jones DB, Schneider BE, et al. Patient race and the likelihood of undergoing bariatric surgery among patients seeking surgery. Surg Endosc. 2015;29(9):2794-9.

17. Edwards MA, Mazzei M, Zhao H, et al. Racial disparities in inferior vena cava filter use in metabolic and bariatric surgery patients: Nationwide insights from the MBSAQIP database. In Press. Am J Surg. 2020;S0002-9610(20)30157-4. https://doi.org/10.1016/j. amjsurg.2020.02.062

18. Crawford AG, Cote C, Couto J, et al. Prevalence of obesity, type II diabetes mellitus, hyperlipidemia, and hypertension in the United States: findings from the GE Centricity Electronic Medical Record database. Popul Health Manag. 2010;13(3):151-61.

19. Nunez Lopez O, Jupiter DC, Bohanon FJ, et al. Health disparities in adolescent bariatric surgery: nationwide outcomes and utilization. J Adolesc Health. 2017;61(5):649-56.

20. Agarwal S, Bruff A, Mazzei M, et al. Exploring racial disparity in perioperative outcomes following revisional bariatric surgery: a case-control matched analysis. Am J Surg. 2020;S00029610(20)30186-0. https://doi.org/10.1016/j.amjsurg.2020.03.030

21. Corral I, Landrine H. Racial differences in the predictors of interest in bariatric surgery in the rural, southeastern USA. J Racial Ethn Health Disparities. 2019;6(3):481-6.

22. Lewis KH, Edwards-Hampton SA, Ard JD. Disparities in treatment uptake and outcomes of patients with obesity in the USA. Curr Obes Rep. 2016;5(2):282-90.

23. Masrur M, Bustos R, Sanchez-Johnsen L, et al. Factors associated with weight loss after metabolic surgery in a multiethnic sample of 1012 patients. Obes Surg. 2020;30(3):975-81.

24. Byrd AS, Toth AT, Stanford FC. Racial disparities in obesity treatment. Curr Obes Rep. 2018;7(2):130-8.

25. Perez NP, Westfal ML, Stapleton SM, et al. Beyond insurance: race-based disparities in the use of metabolic and bariatric surgery for the management of severe pediatric obesity. Surg Obes Relat Dis. 2020;16(3):414-9.

26. Miller-Matero LR, Tobin ET, Clark S, et al. Pursuing bariatric surgery in an urban area: gender and racial disparities and risk for psychiatric symptoms. Obes Res Clin Pract. 2016;10(1):56-62.

27. Wallace AE, Young-Xu Y, Hartley D, et al. Racial, socioeconomic, and rural-urban disparities in obesity-related bariatric surgery. Obes Surg. 2010;20(10):1354-60.

28. Birkmeyer NJ, Gu N. Race, socioeconomic status, and the use of bariatric surgery in Michigan. Obes Surg. 2012;22(2):259-65.

29. Johnson-Mann C, Martin AN, Williams MD, et al. Investigating racial disparities in bariatric surgery referrals. Surg Obes Relat Dis. 2019;15(4):615-20.

30. Gould KM, Zeymo A, Chan KS, et al. Bariatric surgery among vulnerable populations: the effect of the Affordable Care Act's Medicaid expansion. Surgery. 2019;166(5):820-8.

31. de Jager E et al. Disparities in surgical access: a systematic literature review, conceptual model, and evidence map. J Am Coll Surg. 2019;228(3):276-98.

Publisher's Note Springer Nature remains neutral with regard to jurisdictional claims in published maps and institutional affiliations. 